\title{
SUPERFIELD QUANTIZATION OF GENERAL GAUGE THEORIES
}

\author{
P.M.LAVROV申 \\ Tomsk State Pedagogical University, Tomsk, 634041, Russia
}

\begin{abstract}
A superfield version on superspace $\left(x^{\mu}, \theta^{a}\right)$ is proposed for the $S p(2)$ - covariant Lagrangian quantization of general gauge theories. The BRST- and antiBRSTtransformations are realized on superfields as supertranslations in the $\theta^{a}$ - directions. A new (geometric) interpretation of the Ward identities in the quantum gauge theory is given.
\end{abstract}

\section{INTRODUCTION}

Recently, it has been realized [1-8] that BRST-antiBRST invariance can be used as fundamental principle in the construction of quantum gauge theories. This principle has been applied in [1-3] to quantization of dynamical systems with constraints in the Hamiltonian formalism. The $S p(2)-$ covariant Lagrangian quantization of general gauge theories has been developed in [4-6] on the basis of the BRST- antiBRST symmetry principle. Note that in [7] a slightly different (but completely equivalent to $[4,5]$ ) procedure of the $S p(2)-$ covariant Lagrangian quantization was suggested. A completely anticanonical form of the $S p(2)$ - covariant Lagrangian quantization scheme (triplectic quantization in terms of [9]) has been given in [8].

\footnotetext{
${ }^{1}$ E-mail address: lavrov@tspi.tomsk.su
} 
Geometric contents of the BRST- and antiBRST- transformations for the Yang-Mills theories as supertranslations in superspace along additional (Grassmann) coordinates has been realized quite a long time ago [10-15], but there has not been found any satisfactory superfield description of the quantization procedure. Moreover, the crucial point of the superfield descriptions [10-15] was the manifest structure of the Yang-Mills theory action, the problem of treatment of arbitrary gauge theories has remained unsolved.

The main purpose of this paper is to give the superfield quantization rules for general gauge theories, based on the principle of invariance under the BRSTand antiBRST- transformations acting in superspace as supertranslations along the additional (with respect to the space-time coordinates) variables.

The paper is organized as follows. In section 2 the basic objects of the superfield quantization are introduced: superfields $\Phi^{A}(\theta)$, supersources $\bar{\Phi}_{A}(\theta)$, super-antibrackets $(,)^{a}$, operators $\Delta^{a}, V^{a}$; besides some properties of the objects introduced are studied. In section 3 the superfield quantization rules for general gauge theories are formulated. The generating functional of Green functions is constructed as a functional depending on supersources $\bar{\Phi}_{A}$. The superfield form of the BRST- and antiBRST- transformations is found. Independence of the $S$ - matrix on a choice of the gauge is proved. The Ward identities in terms of superfields are derived. In section 4 a connection between the scheme in question and the triplectic $[8,9]$ as well as the $S p(2)$ - covariant [4-6] quantization methods is established, the latter being some particular cases of the superfield quantization, corresponding to special choices of both the solutions of superfield generating equations and the gauge. In section 5 the concluding remarks are made. 
In the paper the condensed notations by DeWitt [16] are used. The Grassmann parity of a quantity $A$ is denoted $\varepsilon(A)$. Derivatives with respect to (super)fields are understood as the right-hand and those with respect to antifields and (super)sources as the left-hand ones. The left derivatives with respect to (super)fields are labelled "l": $\delta_{l} / \delta \phi^{A}$. Scalar anticommuting coordinates $\theta^{a}$ form an $S p(2)$ - doublet. Lowering the $S p(2)$ - indices is given by the rule $\theta_{a}=\varepsilon_{a b} \theta^{b}$. Derivatives with respect to $\theta^{a}$ are understood as the left-hand ones. Integration over $\theta^{a}$ is given by

$$
\int d^{2} \theta=0, \quad \int d^{2} \theta \theta^{a}=0, \int d^{2} \theta \theta^{a} \theta^{b}=\varepsilon^{a b} .
$$

For any function $f(\theta)$ the equalities hold

$$
\int d^{2} \theta \frac{\partial f(\theta)}{\partial \theta^{a}}=0
$$

Any function of $\theta$ can be represented in the form

$$
f(\theta)=f_{o}+f_{a} \theta^{a}+\frac{1}{2} f_{3} \theta_{a} \theta^{a} .
$$

\section{MAIN DEFINITIONS}

Let us introduce a superspace with coordinates $\left(x^{\mu}, \theta^{a}\right)$, where $x^{\mu}(\mu=$ $0,1, \ldots, d-1)$ are the space-time coordinates and $\theta^{a}(a=1,2)$ are anticommuting scalar coordinates. Let $\Phi^{A}(\theta), \varepsilon\left(\Phi^{A}(\theta)\right) \equiv \varepsilon_{A}$ be a set of superfields with the following restriction

$$
\left.\Phi^{A}(\theta)\right|_{\theta=0}=\phi^{A}
$$

where $\phi^{A}$ are the fields of configuration space in the $S p(2)$ - covariant Lagrangian quantization [4-6]. We associate with each superfield $\Phi^{A}(\theta)$ one supersource $\bar{\Phi}_{A}(\theta)$ of the same Grassmann parity,

$$
\varepsilon\left(\bar{\Phi}_{A}(\theta)\right)=\varepsilon_{A} .
$$


In terms of supervariables $\Phi^{A}(\theta), \bar{\Phi}_{A}(\theta)$ we define for any functionals $F=$ $F(\Phi, \bar{\Phi}), G=G(\Phi, \bar{\Phi})$ the super-antibrackets

$$
\begin{aligned}
(F, G)^{a}= & \int d^{2} \theta\left\{\frac{\delta F}{\delta \Phi^{A}(\theta)}(-1)^{\varepsilon_{A}+1} \frac{\partial}{\partial \theta_{a}} \frac{\delta G}{\delta \bar{\Phi}_{A}(\theta)}-\right. \\
& \left.(F \leftrightarrow G)(-1)^{(\varepsilon(F)+1)(\varepsilon(G)+1)}\right\}
\end{aligned}
$$

with the evident property of generalized antisymmetry

$$
(F, G)^{a}=-(-1)^{(\varepsilon(F)+1)(\varepsilon(G)+1)}(G, F)^{a} .
$$

The super-antibrackets (3) satisfy the generalized Jacobi identities

$$
\left((F, G)^{\{a}, H\right)^{b\}}(-1)^{(\varepsilon(F)+1)(\varepsilon(H)+1)}+\operatorname{cycle}(F, G, H) \equiv 0,
$$

where the curly bracket denotes symmetrization of $a$ and $b$.

Let us introduce the operators $\Delta^{a}$ and $V^{a}$ by the rule

$$
\begin{gathered}
\Delta^{a}=-\int d^{2} \theta \frac{\delta_{l}}{\delta \Phi^{A}(\theta)} \frac{\partial}{\partial \theta_{a}} \frac{\delta}{\delta \bar{\Phi}_{A}(\theta)} \\
V^{a}=\int d^{2} \theta\left(\frac{\partial \bar{\Phi}_{A}(\theta)}{\partial \theta_{a}} \frac{\delta}{\delta \bar{\Phi}_{A}(\theta)}+\frac{\partial \Phi^{A}(\theta)}{\partial \theta_{a}} \frac{\delta_{l}}{\delta \Phi^{A}(\theta)}\right) .
\end{gathered}
$$

One may easily check that the following properties hold

$$
\begin{gathered}
\Delta^{\{a} \Delta^{b\}}=0, \\
V^{\{a} V^{b\}}=0, \\
\Delta^{a} V^{b}+V^{b} \Delta^{a}=0 .
\end{gathered}
$$

The action of the operators $\Delta^{a}$ and $V^{a}$ upon the super- antibrackets is given by the relations

$$
\Delta^{\{a}(F, G)^{b\}}=\left(\Delta^{\{a} F, G\right)^{b\}}-\left(F, \Delta^{\{a} G\right)^{b\}}(-1)^{\varepsilon(F)},
$$




$$
V^{a}(F, G)^{b}=\left(V^{a} F, G\right)^{b}-\left(F, V^{a} G\right)^{b}(-1)^{\varepsilon(F)} .
$$

It is also convinient to introduce the extended operators $\bar{\Delta}^{a}$

$$
\bar{\Delta}^{a}=\Delta^{a}+\frac{i}{\hbar} V^{a}
$$

These operators satisfy the relations

$$
\bar{\Delta}^{\{a} \bar{\Delta}^{b\}}=0
$$

which follow from (8)-(10).

\section{SUPERFIELD QUANTIZATION}

The basic object of superfield quantization is the quantum action $W=$ $W(\Phi, \bar{\Phi})$. We require that $W$ be a solution to the following generating equations (quantum master equations)

$$
\bar{\Delta}^{a} \exp \left\{\frac{i}{\hbar} W\right\}=0
$$

or equivalently

$$
\frac{1}{2}(W, W)^{a}+V^{a} W=i \hbar \Delta^{a} W .
$$

The generating functional of the Green functions $Z=Z(\bar{\Phi})$ for superfields $\Phi^{A}(\theta)$ we define as

$$
\begin{aligned}
Z(\bar{\Phi})= & \int\left[d \Phi^{\prime}\right]\left[d \bar{\Phi}^{\prime}\right] \rho\left(\bar{\Phi}^{\prime}\right) \exp \left\{\frac { i } { \hbar } \left[W\left(\Phi^{\prime}, \bar{\Phi}^{\prime}\right)-\right.\right. \\
& \left.\left.-\frac{1}{2} \varepsilon_{a b} V^{\prime a} V^{\prime b} F\left(\Phi^{\prime}\right)-\bar{\Phi} \Phi^{\prime}\right]\right\},
\end{aligned}
$$

where $F(\Phi)$ is the boson gauge functional, $\rho(\bar{\Phi})$ is the weight functional having the form of functional $\delta$ - function

$$
\rho(\bar{\Phi})=\delta\left(\int d^{2} \theta \bar{\Phi}(\theta)\right)
$$


and the notation

$$
\bar{\Phi} \Phi \equiv \int d^{2} \theta \bar{\Phi}_{A}(\theta) \Phi^{A}(\theta)
$$

is used.

The introduced above generating functional (17) possesses two important properties. Firstly, the integrand in (17) for $\bar{\Phi}=0$ is invariant under the transformations of global supersymmetry

$$
\begin{gathered}
\delta \Phi^{A}(\theta)=\mu_{a} \frac{\partial \Phi^{A}(\theta)}{\partial \theta_{a}}, \quad \varepsilon\left(\mu_{a}\right)=1, \\
\delta \bar{\Phi}_{A}(\theta)=\mu_{a} \frac{\partial \bar{\Phi}_{A}(\theta)}{\partial \theta_{a}}+\mu_{a} \frac{\partial}{\partial \theta_{a}} \frac{\delta W}{\delta \Phi^{A}(\theta)}
\end{gathered}
$$

on account of the generating equations (16) and the invariance of weight functional (18) under transformations (21)

$$
\delta \rho(\bar{\Phi})=0
$$

In $(20),(21) \mu_{a}$ is a $S p(2)$ - doublet of the constant anticommuting Grassmann parameters. Secondly, the vacuum functional $Z(0)$ does not depend on choice of the gauge boson $F$ within the proposed superfield scheme (16)-(18). Indeed, suppose $Z_{F} \equiv Z(0)$. We shall change the gauge $F(\Phi) \rightarrow F(\Phi)+\delta F(\Phi)$. In the functional integral for $Z_{F+\delta F}$ we make the change of variables (20),(21) choosing for the parameters $\mu_{a}$

$$
\mu_{a}=-\frac{i}{2 \hbar} \varepsilon_{a b} V^{b} \delta F(\Phi)
$$

Taking into account (9),(16) and (22) we find that

$$
Z_{F+\delta F}=Z_{F}
$$

and, hence, the $S$ - matrix is gauge-invariant. 
The transformations (20), (21) realize the BRST- and antiBRST- symmetry in the superfield approach to quantum gauge theory. Allowing for (3),(7) one can rewrite these transformations in the form

$$
\begin{gathered}
\delta \Phi^{A}(\theta)=\mu_{a} V^{a} \Phi^{A}(\theta), \\
\delta \bar{\Phi}_{A}(\theta)=\mu_{a} V^{a} \bar{\Phi}_{A}(\theta)+\mu_{a}\left(W, \bar{\Phi}_{A}(\theta)\right)^{a} .
\end{gathered}
$$

From (25), (26) we conclude that the operators $V^{a}(7)$ can be considered as generators of supertranslations in the $\theta^{a}$ - directions on supervariables $\Phi^{A}(\theta), \bar{\Phi}_{A}(\theta)$. This gives a geometric interpretation of the BRST- and antiBRST- symmetry for arbitrary gauge theory.

Invariance of vacuum functional $Z(0)$ under the BRST- and antiBRSTtransfomations leads to the presence of gauge Ward identities. Let us consider the derivation of these identities. To do this we shall use the standard assumptions on functional integral properties, in particular,

$$
\int[d \Phi][d \bar{\Phi}] \rho(\bar{\Phi}) \frac{\delta F(\Phi, \bar{\Phi})}{\delta \Phi}=0, \int d[\Phi][d \bar{\Phi}] \rho(\bar{\Phi}) \frac{\delta F(\Phi, \bar{\Phi})}{\delta \bar{\Phi}}=0 .
$$

Taking into account the explicit form of the operators $\bar{\Delta}^{a}$ and (27) we have the following equalities

$$
\begin{gathered}
\int\left[d \Phi^{\prime}\right]\left[d \bar{\Phi}^{\prime}\right] \rho\left(\bar{\Phi}^{\prime}\right) \bar{\Delta}^{\prime a} \exp \left\{\frac { i } { \hbar } \left[W\left(\Phi^{\prime}, \bar{\Phi}^{\prime}\right)-\right.\right. \\
\left.\left.-\frac{1}{2} \varepsilon_{a b} V^{\prime a} V^{\prime b} F\left(\Phi^{\prime}\right)-\bar{\Phi} \Phi^{\prime}\right]\right\}=0
\end{gathered}
$$

Let us act on exponential by the operators $\bar{\Delta}^{a}$ and take into account (9), (10), (16). We obtain

$$
V^{a} Z(\bar{\Phi})=0 .
$$

Equations (29) represent the superfield form of Ward identities for generating functional of Green functions. 
Let us now consider the Legendre transformation of $\ln Z(\bar{\Phi})$ with respect to the supersources $\bar{\Phi}_{A}(\theta)$. For this purpose we define superfields $\Phi^{A}(\theta)$ by the relations

$$
\Phi^{A}(\theta)=-\frac{\hbar}{i} \frac{\delta \ln Z(\bar{\Phi})}{\delta \bar{\Phi}_{A}(\theta)}
$$

and introduce the functional $\Gamma(\Phi)$ by the rule

$$
\Gamma(\Phi)=\frac{\hbar}{i} \ln Z(\bar{\Phi})+\bar{\Phi} \Phi
$$

so that

$$
\frac{\delta \Gamma}{\delta \Phi^{A}(\theta)}=\bar{\Phi}_{A}(\theta)
$$

$\Gamma(\Phi)$ is the vertex functions generating functional. Rewriting the Ward identities (29) for the vertex functios generating functional, we have the relations

$$
V^{a} \Gamma(\Phi)=0
$$

as the superfield form of the Ward identities for $\Gamma(\Phi)$.

From (29), (33) one can establish a new (geometric) interpretation of the Ward identities in quantum gauge theory. Indeed, the Ward identities express the invariance of the generating functionals $Z$ and $\Gamma$ under supertranslations in the $\theta^{a}$ - directions.

\section{CONNECTION WITH THE TRIPLECTIC AND $S p(2)-$ COVARIANT QUANTIZATIONS}

It is useful to compare the suggested superfield quantization for general gauge theories with the $S p(2)$ - covariant Lagrangian quantization [4-6] and the triplectic one [8]. To this end we present, first of all, the matter of developed superfield quantization scheme in the component form. 
For supervariables $\Phi^{A}(\theta)$ and $\bar{\Phi}_{A}(\theta)$ we shall use the following notations in the $\theta^{a}$ - expansions

$$
\begin{aligned}
& \Phi^{A}(\theta)=\phi^{A}+\pi^{A a} \theta_{a}+\frac{1}{2} \lambda^{A} \theta_{a} \theta^{a}, \\
& \bar{\Phi}_{A}(\theta)=\bar{\phi}_{A}-\theta^{a} \phi_{A a}^{*}-\frac{1}{2} \theta_{a} \theta^{a} J_{A} .
\end{aligned}
$$

Then the operators $\Delta^{a}(6), V^{a}(7)$ and super-antibrackets (3) have the form

$$
\begin{gathered}
(F, G)^{a}=\frac{\delta F}{\delta \phi^{A}} \frac{\delta G}{\delta \phi_{A a}^{*}}+\varepsilon^{a b} \frac{\delta F}{\delta \pi^{A b}} \frac{\delta G}{\delta \bar{\Phi}_{A}}- \\
(F \leftrightarrow G)(-1)^{(\varepsilon(F)+1)(\varepsilon(G)+1)}, \\
\Delta^{a}=(-1)^{\varepsilon_{A}} \frac{\delta_{l}}{\delta \phi^{A}} \frac{\delta}{\delta \phi_{A a}^{*}}+(-1)^{\varepsilon_{A}+1} \varepsilon^{a b} \frac{\delta_{l}}{\delta \pi^{A b}} \frac{\delta}{\delta \bar{\phi}_{A}}, \\
V^{a}=\varepsilon^{a b} \phi_{A b}^{*} \frac{\delta}{\delta \bar{\phi}_{A}}-(-1)^{\varepsilon_{A}} \pi^{A a} \frac{\delta_{l}}{\delta \phi^{A}}+ \\
+(-1)^{\varepsilon_{A}} \varepsilon^{a b} \lambda^{A} \frac{\delta_{l}}{\delta \pi^{A b}}-J_{A} \frac{\delta}{\delta \phi_{A a}^{*}} .
\end{gathered}
$$

In the component form the gauge fixing action reads

$$
\frac{1}{2} \varepsilon_{a b} V^{a} V^{b} F(\Phi)=\frac{1}{2} \varepsilon_{a b} \pi^{A a} \frac{\delta^{2} F}{\delta \phi^{A} \delta \phi^{B}} \pi^{B b}+\frac{\delta F}{\delta \phi^{A}} \lambda^{A}-
$$

$$
-\frac{1}{2} \varepsilon^{a b} \lambda^{A} \frac{\delta^{2} F}{\delta \pi^{A a} \delta \pi^{B b}} \lambda^{B}+\pi^{A a} \frac{\delta^{2} F}{\delta \phi^{A} \delta \pi^{B a}} \lambda^{B},
$$

For the functional $\bar{\Phi} \Phi$ in (17) we have

$$
\bar{\Phi} \Phi=\bar{\phi}_{A} \lambda^{A}+\phi_{A a}^{*} \pi^{A a}-J_{A} \phi^{A}
$$

Integration measure

$$
[d \Phi][d \bar{\Phi}] \rho(\bar{\Phi})=[d \phi]\left[d \phi^{*}\right][d \pi][d \bar{\phi}][d \lambda][d J] \delta(J)
$$

coincides, in fact, with measures in functional integrals of papers [4-6] and [8].

Let us now turn ourselves to the generating equations (16) of the superfield formalism. By virtue of (36)-(38) we can see that the variables $\lambda^{A}$ and $J_{A}$ 
enter the equations (16) in a very special manner, being only contained in the operators $V^{a}$ as linear multipliers at the differential operators. We shall make use of this special point. To this end, we note that the functional $\bar{\Phi} \Phi$ is linear with respect to $\lambda^{A}, J_{A}$ and satisfies both the equations

$$
V^{a} \bar{\Phi} \Phi=0
$$

and (16) as a whole. This enables one to seek solutions of the equations (16) in the form

$$
W(\Phi, \bar{\Phi})=S(\Phi, \bar{\Phi})+\bar{\Phi} \Phi
$$

with the functional $S(\Phi, \bar{\Phi})$ subjected to the conditions

$$
\begin{gathered}
\int d^{2} \theta \frac{1}{2} \theta_{a} \theta^{a} \frac{\delta S}{\delta \Phi^{A}(\theta)}=\frac{\delta S}{\delta \lambda^{A}}=0 \\
\int d^{2} \theta \frac{1}{2} \theta_{a} \theta^{a} \frac{\delta S}{\delta \bar{\Phi}_{A}(\theta)}=-\frac{\delta S}{\delta J_{A}}=0 .
\end{gathered}
$$

Taking (43) and (36)-(38) into account, we find that the functional $S$ is exactly a solution to the generating equations of paper [8]. Consequently, the special choice of solutions to the equations (16) in the form (42),(43) with $\bar{\Phi}=0$ leads to the vacuum functional of paper [14], corresponding to the special choice of the gauge (note that in [14], apart from a particular choice of gauge fixing, the general approach to solution of this problem is studied).

One can also readily establish a connection with the $S p(2)$ - covariant quantization of papers [4-6]. To do this, suffice it to require, in addition, that the functional $S$ be independent on the variables $\pi^{A a}$, i.e.

$$
\frac{\delta S}{\delta \pi^{A a}}=0
$$

choosing the gauge fixing functional $F$ as to depend on the variables $\phi^{A}$ only: $F=F(\phi)$. Then we find in (17) the exact form of the generating functional of Green functions in the $S p(2)$ - covariant quantization of papers [4-6]. 
The form (29) of the Ward identities for $Z(\bar{\Phi})$, rewritten in terms of the components

$$
J_{A} \frac{\delta Z}{\delta \phi_{A a}^{*}}-\varepsilon^{a b} \phi_{A b}^{*} \frac{\delta Z}{\delta \bar{\phi}_{A}}=0
$$

coincides with the one, derived in [4].

The component form of the definition (30) for $\Phi^{A}(\theta)$ reads

$$
\phi^{A}=\frac{\hbar}{i} \frac{\delta \ln Z}{\delta J_{A}}, \quad \pi^{A a}=\frac{\hbar}{i} \frac{\delta \ln Z}{\delta \phi_{A a}^{*}}, \quad \lambda^{A}=\frac{\hbar}{i} \frac{\delta \ln Z}{\delta \bar{\phi}_{A}},
$$

Given this, the equations (32) can be written as

$$
\frac{\delta \Gamma}{\delta \phi^{A}}=-J_{A}, \quad \frac{\delta \Gamma}{\delta \pi^{A a}}=\phi_{A a}^{*}, \quad \frac{\delta \Gamma}{\delta \lambda^{A}}=\bar{\phi}_{A} .
$$

For the Ward identities (34) we have the following equations

$$
\pi^{A a} \frac{\delta \Gamma}{\delta \phi^{A}}+\varepsilon^{a b} \lambda^{A} \frac{\delta \Gamma}{\delta \pi^{A b}}=0
$$

The form (45) of the Ward identities for the generating functional of vertex functions $\Gamma$ differs from the one, obtained in [4]. The difference is due to the fact that in [4] the transition from the Ward identities for $Z$ (coinciding, formally, with (44)) to the ones for $\Gamma$ was made by the Legendre transformation with respect to the variables $J_{A}$ only, the variables $\phi_{A a}^{*}, \bar{\phi}_{A}$ being inert.

\section{DISCUSSION}

In this paper a closed superfield representation of the $S p(2)$ - covariant quantization [4-6] for gauge theories is found. One of the basic objects of the method in question is a superfield $\Phi^{A}(\theta)$ subject to the boundary condition $\left.\Phi^{A}(\theta)\right|_{\theta=0}=\phi^{A}$, where $\phi^{A}$ is the set of fields of configuration space in the $S p(2)$-covariant quantization formalism [4-6]. To each superfield $\Phi^{A}(\theta)$ there is 
assigned the corresponding supersource $\bar{\Phi}_{A}(\theta)$ with the same Grassmann parity. The variables belonging to the complete set of the $S p(2)$ - covariant quantization method arise in the superfield formalism quite naturally as the corresponding components of all the supervariables $\Phi^{A}(\theta), \bar{\Phi}_{A}(\theta)$ (see $(34),(35)$ ).

The BRST-antiBRST symmetry transformations (20),(21) (or, equivalently $(25),(26))$ for arbitrary gauge theories have within the superfield formalism a clear geometric contents as they are realized as supertranslations in superspace $\left(x^{\mu}, \theta^{a}\right)$ along the Grassmann coordinates $\theta^{a}$.

The superfield description permits one to have a new look at the Ward identities in the quantum theory of gauge fields, thus revealing their geometric contents. Indeed, the identities (29) and (33) for both the generating functional of Green functions $Z=Z(\bar{\Phi})$ and the generating functional of vertex functions $\Gamma=\Gamma(\Phi)$ are nothing but the statements that $Z$ and $\Gamma$ are invariant under supertranslations in superspace. Simultaneously, the role and geometric origin of the operators $V^{a}$, realizing the supertranslations in terms of the variables $\Phi^{A}(\theta), \bar{\Phi}_{A}(\theta)$ are revealed.

The superfield description permits a universal outlook on the quantization scheme for general gauge theories, developed in papers [4-6] and [8,9]; the latter are the particular cases of the superfield description as they correspond to certain special choices of both the solutions to the generating equations (16) and the gauge.

\section{Acknowledgement}

The author is grateful to I.V.Tyutin and V.I.Mudruk for helpful disscussions. This work has been supported in part by grant RI 1300 from the International 
Science Foundation and by the Russian Fund for Fundamental studies under grant number 94-02-03234. 


\section{References}

[1] Batalin I.A., Lavrov P.M., Tyutin I.V., J. Math. Phys. 31 (1990) 6.

[2] Batalin I.A., Lavrov P.M., Tyutin I.V., J. Math. Phys. 31 (1990) 2708.

[3] Batalin I.A., Lavrov P.M., Tyutin I.V., Int. J. Mod. Phys. 6 (1991) 3599.

[4] Batalin I.A., Lavrov P.M., Tyutin I.V., J. Math. Phys. 31 (1990) 1487.

[5] Batalin I.A., Lavrov P.M., Tyutin I.V., J. Math. Phys. 32 (1991) 532.

[6] Batalin I.A., Lavrov P.M., Tyutin I.V., J. Math. Phys. 32 (1991) 2513.

[7] Hull C.M., Mod. Phys. Lett. A5 (1991) 1871.

[8] Batalin I., Marnelius R., Göteborg preprint ITP 94-30 (1995).

[9] Batalin I.A., Marnelius R., Semikhatov A.M., Göteborg preprint ITP 9431 (1995).

[10] Bonora L., Tonin M., Phys. Lett. B98 (1981) 48.

[11] Bonora L., Pasti P., Tonin M., J. Math. Phys. 23 (1982) 839.

[12] Hoyos J. Quiros M., Mittelbrunn J.R., De Urries F., Nucl. Phys. B218 (1983) 159 .

[13] Baulieu L., Nucl. Phys. B218 (1983) 157.

[14] Baulieu L., Phys. Rep. 129 (1985) 1.

[15] Hull C.M., Spence B., Vazquez-Bello J.L., Nucl. Phys. B348 (1991) 108.

[16] De Witt B.S., Dynamical Theory of Groups and Fields (Gordon and Breach, 1965). 